\title{
First Offensive Shot in Elite Table Tennis
}

\section{El primer golpe ofensivo en el tenis de mesa de élite}

\author{
Michael Fuchs, $1,2,3 *$; Martin Lames 1 \\ 1 Technical University of Munich, Munich, Germany \\ 2 International Table Tennis Federation, Lausanne, Switzerland \\ 3 National Paralympic Committee Germany
}

\section{Received: 18-2-2021}

Accepted: 21-7-2021

\begin{abstract}
From the very first shots in table tennis, players face a basic tactical decision: either return the ball short and defensively or attack it with an offensive shot. Thus, the real turntable of a rally is the shot - in this study called "first offensive shot" (FOS) - which is the transition from defensive to offensive play. This study investigates the FOS, which is defined as the first shot after the serve without any kind of backspin/side-backspin, for 90 matches (nrally $=7449$ ) of the 2016 Rio Olympic Games. The FOS parameters - laterality, technique and position to the table at the point of contact - and the respective winning probabilities are analysed. The influences of sex and the players' ranking (resulting in three different match categories) on those parameters are studied. Descriptive statistics about the incidences of the FOSs show that four typical FOSs cover $98 \%$ of all FOSs. Chi-square tests reveal a significant relation between sex and these typical FOSs. Regarding the match categories, the tests prove a significant relation between match categories and FOS tactics for both genders. A difference in the FOS tactics between the serving and the receiving player is found as well. The winning probabilities show that using topspin (Forehand and Backhand) as FOS was an advantage in every match category, whereas using flip as FOS led mostly to a winning probability below $50 \%$ for the FOS player. Compared to prevailing methods in table tennis match analysis, which are based on fixed shot numbers, taking the FOS as object of analyses of rally opening is an innovative new approach focusing on the tactical meaning of shots that is not expressed in shot number.
\end{abstract}

Keywords: First offensive shot, racket sports, table tennis, match analysis.

\section{Resumen}

En los primeros golpes del tenis de mesa, los jugadores se enfrentan a una decisión táctica básica: devolver la pelota en corto y de forma pasiva o atacarla con un golpe ofensivo. En el primer caso, hay menos riesgo, pero no hay presión para hacer el punto; en el segundo caso, se crea presión, pero con un alto riesgo porque el servicio y los previos golpes "pasivos" tratan de dificultar al máximo un golpe ofensivo, que normalmente es corto y plano. Por lo tanto, el verdadero punto de inflexión de un peloteo es este golpe -en este estudio llamado “primer golpe ofensivo" (FOS, por su sigla en inglés)-, el cual es la transición del juego pasivo al ofensivo. Este estudio investiga el FOS, el cual se define como el primer golpe después del servicio sin ponerle efecto a la pelota, para 90 partidos $\left(n_{\text {rally }}=7449\right)$ de los Juegos Olímpicos de Río 2016. Se analizan los parámetros del FOS -lateralidad, técnica y posición en la mesa en el punto de contacto- y las respectivas probabilidades de victoria. Se estudian las influencias del género y de la clasificación de los jugadores (lo que da lugar a tres categorías de partidos diferentes) en esos parámetros. La estadística descriptiva sobre las incidencias de los FOS muestra que cuatro FOS típicos cubren el 98

Correspondence author: Michael Fuchs, michael.fuchs@mytum.de

Cite this article as:

Fuchs, M., \& Lames, M. (2021). First Offensive Shot in Elite Table Tennis. International Journal of Racket Sports Science, 3(1), 10-21.

This is an open access article under the CC BY license (https://creativecommons.org/licenses/by/4.0/). 
$\%$ de todos los FOS. Las pruebas $x^{2}$ revelan una relación significativa entre el género y estos FOS típicos. En cuanto a las categorías de los partidos, las pruebas demuestran una relación significativa entre las categorías de los partidos y la táctica del FOS para ambos géneros. También se encontró una diferencia en la táctica del FOS entre el jugador que sirve y el que recibe. Las probabilidades de victoria muestran que usar el efecto (de derecha o de revés) como FOS fue una ventaja en todas las categorías de los partidos, mientras que el uso del flip como FOS condujo en su mayoría a una probabilidad de victoria inferior al 50 \% para el jugador del FOS. Excepto por el flip derecho en los partidos femeninos, siempre fue una mayor ventaja para el jugador del FOS si este podía terminar el peloteo de último con el ataque subsiguiente después del FOS, lo que significa que la probabilidad de victoria para el jugador del FOS disminuyó en los peloteos más largos. En comparación con los métodos predominantes en el análisis de partidos de tenis de mesa, que se basan en los números fijos de golpes, tomar el Fos como objeto de análisis del inicio del peloteo es un innovador y nuevo enfoque que se centra en el significado táctico de los golpes que no se expresa en el número de golpes

Palabras clave: Primer golpe ofensivo, deportes de raqueta, tenis de mesa, análisis de partidos.

\section{Introduction}

After the beginning of the sport in the second half of the $19^{\text {th }}$ century, table tennis has progressed enormously. Not only the International Table Tennis Federation (ITTF) has been growing to the sports federation with the highest number of members in terms of national associations (226), but also the sport itself has been always going through developments in different areas. Due to different rule changes and technological developments, the material of the players developed as well. Big milestones in this area were the invention of the sponge rubber in the 1950s, the invention of the speed glue in the $1970 \mathrm{~s}$, the change from $38 \mathrm{~mm}$ to $40 \mathrm{~mm}$ ball diameter in 2000 , and latest the ban of the speed glue with volatile organic compounds (VOCs) as well as the introduction of the celluloidfree balls in 2014 (Clemett, 2010; Küneth, 2020).

The players did not only adapt their material over the years, but also their way of playing from changing to a more spin-oriented game style with the invention of the sponge rubber in the 1950s ending up with new shot techniques called "Strawberry" or "Chiquita" in recent years. Until the late 2000 s it seemed to be normal to play the short game until the push of one player gets long enough to attack with topspin. But especially since Zhang Jike (World and Olympic Champion) trademarked the sidespin-topspin backhand flip the so called "Chiquita" - and made it popular, more and more players seem to leave the short game early using this technique which has developed quite fast in recent years (Townsend, 2017). With this specific technique which is used by players not only in backhand side, but also in the forehand side, players can get quite easily out of a rather defensive short game into the offensive game.
This transition from the defensive short game to the offensive attacking game is a very crucial and decisive tactical decision in almost each rally in table tennis: On one hand a player can gain advantage putting the opponent in a defensive position by attacking first, on the other hand, as the first offensive technique in a rally, we call it "first offensive shot" (FOS) is technically difficult and has mostly to be played against a short ball and/or a ball with some backspin played with the intention of not allowing for an offensive shot, the FOS is a risky shot. Besides the risk of a direct error, there is the risk of a FOS of too low quality, so that it can be countered immediately and successfully.

The FOS might be seen as a rewarding technique, if effectively executed, but rather disadvantageous when not being played with high quality. Thus, playing the FOS is a basic tactical decision in almost each table tennis rally (the only exceptions are the very rare serve winners and errors).

On one hand, this is a situational decision of players in the match dependent on the quality of the serve or prior defensive shot, but on the other hand it is also a tactical element of a match strategy for players and coaches to decide whether to go for the FOS or leave it to the opponent. This decision should be supported by match analysis and data collected on the specific opponent.

In this study a new structural model for a table tennis rally including the FOS and develop a corresponding observational system focusing on recording properties of the FOS was introduced. FOS may not be defined based on a shot number in the rally (like serve, receive, third shot, fourth shot etc.), because it is not known a priori which shot will be the FOS. FOSs are semantically similar shots defined as the first shot in a rally without any kind of backspin (serves excluded). Figure 1 shows the process model of a table tennis rally. 


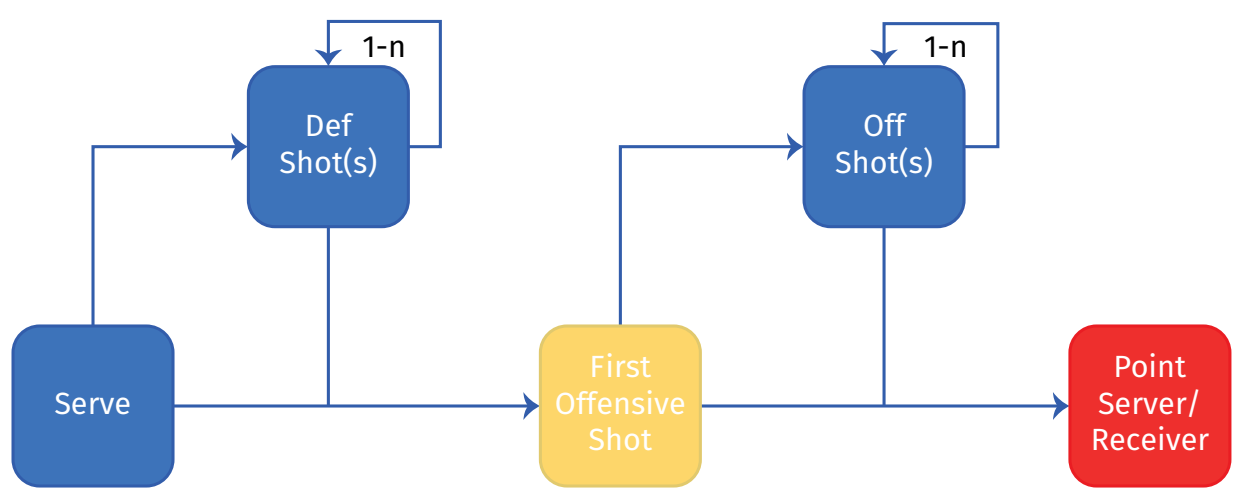

Figure 1. Process model of table tennis rally with first offensive shot.

The model separates the rally in three phases: The first phase consists of serve and defensive shot(s), second and central phase is the FOS, and the third phase contains the following offensive shot(s) until the end of the rally. Accordingly, phase one and three might include a different number of shots in each rally (from 0 to $n$ ) and the shot number of the FOS might vary in each rally. The (rare) occasions of point/error with serve or defensive shot are not depicted.

It must be mentioned that this model does not apply to matches with a defensive player as in this case the FOS is most likely followed by defensive shots of the defensive player again and the process would be repeated from phase one within a rally. As only $3 \%$ of the current top 100 ranked men and $12 \%$ of the current top 100 ranked women in the world ranking are defenders (in January 2021) (International Table Tennis Federation, 2021) these players/matches are neglected in this study.

Different approaches like notational analysis, footwork analysis, performance indices and simulative approaches have been used in table tennis analysis (Fuchs et al., 2018; Malagoli Lanzoni et al., 2014). By analysing existing literature, regardless the approach of the analysis, the FOS has not been addressed in previous table tennis research. Performance analysis in table tennis is typically based on a shot-number based approach, e.g. the three-phase-method in Japan and China, which gives feedback regarding rally length, winning probabilities and error rates of specific shot numbers (Tamaki et al., 2017; Wu \& Li, 1992; Zhang et al., 2013). The problem with shot-number based approaches is that the shot number itself doesn't reflect necessarily the semantics or meaning of a shot, e.g. shot \#4 may be an all-in attacking forehand topspin shot, a short, defensive backhand push or the first offensive shot in a rally. As a consequence, performance indicators based on shot-number based approaches, e.g. technique effectiveness (Zhang et al., 2013) suffer from this ambiguity. Although this problem has been acknowledged, for example including the techniques used in shot number base approaches, e.g. for shot \#1 (serve) and shot \#2 (receive) (Djokic et al., 2017; Zhang \& Zhou,
2017), the problem remains that semantically similar shots are hard to analyse in these approaches if they have different shot numbers (Zhang \& Zhou, 2017).

The aim of this study is to design an observational system to analyse the following characteristics regarding the FOS in a rally in elite table tennis:

- techniques used for FOSs

- position where the FOSs are performed (over vs. behind the table)

- shot number of the FOSs

- serving or receiving players performs the FOS

- differences between men and women and between top ranked and lower ranked players regarding the FOS behaviour?

- winning probability for the FOS player

\section{Methods}

First offensive shot (FOS) \& prevalent FOS techniques

The FOS in a rally is defined as the first shot after the serve without any kind of backspin/sidebackspin. Thus, the serve itself is excluded by this definition, regardless of its spin. Considering the spin condition with the resulting fact that there is either a serve or a defensive backspin/side-backspin shot prior the FOS, possible techniques for the FOS are Flip (including Chiquita), Topspin, Smash, Drive and Special (any other offensive shots which can't be assigned to the other categories) whereas Smash, Drive and Special are summarized as "other" due to their rare use as FOS.

\section{Position relative to table at the point of contact}

The position relative to the table at the point of contact is defined as the location where the player hits the ball considering the actual shot movement. In our study we distinguish between two possible positions. The first is "over the table", where techniques need to be adapted because the table poses an environmental constraint (Newell, 1986). The second is "behind the table", where the player hits the ball close to the edge or behind the table 
and is not restricted in his/her movement range (especially at the backward movement phase of the shot due to the table. Balls hit after going off the side of the table (and not off the back) and are hit on the side with a full backswing are considered as behind the table shots as well.

\section{Independent variables}

Two independent variables were used in this study. First, matches of male and female players were compared. Second, using the ITTF world ranking list published on August 1st, 2016 (current ranking lists at the 2016 Olympic games), players were divided into two player categories ("top 50" and "over 50"), resulting in three possible match categories ("top 50 vs. top 50", "top 50 vs. over 50" and "over 50 vs. over $50 "$ ). This leads to a two-factor factorial design (sex versus match category).

\section{Data collection and sample}

Matches of the 2016 Olympic Games were analysed using video recordings of the International Table Tennis Federation (ITTF)/International Olympic Committee (IOC). By entering the Olympic Games, participants agree to be filmed, televised, photographed, identified and/or otherwise recorded during the Olympic Games, and that their captured or recorded image, together with their name, likeness, voice, performance and biographical information, may be used in any content, format and through any media or technology whether now existing or created in the future (International Olympic Committee, 2016). All data were recorded in an anonymous dataset. Procedures performed in the study were in strict accordance with the Declaration of Helsinki as well as with the ethical standards of the Technical University of Munich, Germany.Matches with players who have a defensive playing style (choppers) and/or use non-attacking rubbers like long pimples were excluded from the sample as they are expected to bias the FOS statistics due to their nonattacking style as mentioned in the previous chapter. 53 different female players (15 left-handed, 38 righthanded; one with one half-long pimple rubber, six with one short pimple rubber, 46 with two backside rubbers) from 34 countries and 48 different male players (twelve left-handed, 36 right-handed; all with two backside rubbers) from 34 countries are included in the sample. All players were using the shakehand grip.

A total of 90 matches were analysed, including 45 men's and 45 women's matches. 15 matches per match category were chosen. Especially matches with "over 50 " players were limited as only one woman and four men of this category made it into the round of 16 of the singles competition. Thus, all possible matches of the singles competition with "over 50 " players involved were chosen and complemented with matches from the team competition. For the "top50 vs top50" category, matches of the finals and semi-finals of both competitions were analysed and complemented with matches from earlier stages. The 90 analysed matches led to a total number of 7449 analysed rallies, 3889 rallies of men's matches and 3560 rallies of women's matches respectively. Table 1 shows the distribution of matches and rallies according to match categories and sex.

\section{Data analysis and observer agreement}

All matches were analysed with the table tennis video analysis tool "TUM.TT" (Lames et al., 2018). For this study only the FOSs were analysed in the deepanalysis-mode of TUM.TT. Therefore, the observer had to identify if there has been a FOS in the rally and mark this shot. For those marked FOSs, the following parameters were collected additionally: Laterality (Forehand $(\mathrm{FH}) /$ Backhand $(\mathrm{BH})$ ), technique (Flip, Topspin, Smash, Drive and Special) and position relative to table at the point of contact (over/behind the table). Based on the collected variables, three more variables were defined and subject to analyses: FOS by server/receiver, number of shots after FOS and FOS direct impact (yes/no winner/error?).

The inter-observer reliability test calculating an intraclass correlation coefficient (model:two-way mixed, type: absolute, confidence interval=95\%) was conducted using a randomly chosen sample of 6 matches (one from each match category) with 517 rallies. These rallies were analysed by two independent observers (one of the authors and a German B-licenced table tennis coach from a Bundesliga club). Reliability was assessed with a two-step approach: First, the identification of the observational unit, i.e. a shot as a FOS in a rally, was tested and resulted in perfect agreement expressed by an intraclass correlation coefficient of 1.000. Second, agreement of assigning levels of the observed variables was tested. The intraclass correlation coefficients for laterality, technique and position relative to table at the point of contact were 1.000, .957 (Lower 95\% confidence interval (L95\%) at .949) and .940 (L95\% = .920) respectively. Thus, for all recorded variables the inter-rater agreement was excellent (Koo \& Li, 2016).

\section{Statistical Analysis}

IBM SPSS Statistics 25 (IBM Corp., Armonk, New York, USA) was used for the statistical analyses. Descriptive statistics for FOS shot number, laterality-techniqueposition-combination of the FOS are presented in dependence of sex and match category. The shot number of FOS was tested for normal distribution with the Kolmogorov-Smirnov test and proved violations of normality for FOS shot number (heavily right skewed distribution).

Cross tables and chi-square tests with the Monte Carlo method if necessary were used to identify relations between the independent variables (sex, match category) and the dependent (calculated) variables (FOS laterality, FOS technique, FOS by server/ 
receiver). For comparison of the FOS shot number between female and male and the match category groups, Mann-Whitney tests and Kruskal-Wallis tests were conducted.

Alpha was set at 0.05 for all analyses. Correlation coefficient $r$ was used as effect size for the Mann Whitney $\mathrm{U}$ test as well as for the pairwise comparisons of the Kruskal-Wallis-test in case of significance (Cohen, 1988; Fritz et al., 2012).

\section{Results}

From 7449 analysed rallies, 6771 (90.9\%) rallies contained a FOS. In $668(9.0 \%)$ rallies there was no FOS because of prior rally termination (serve winner (6.0\%), serve error (13.8\%), defensive shot winner (17.5\%) and defensive shot error (62.7\%)). Ten (0.1\%) rallies had to be excluded from the sample as the (potential) FOS was not visible in the video footage due to replays or a blocked view.

Four laterality-technique-position combinations (out of twelve) cover $98.3 \%$ of all 6771 analysed FOSs. Therefore, we excluded the other eight categories from subsequent analysis. The descriptive statistics of our two-factor factorial model regarding these four laterality-technique-position combinations are shown in Table 2.

The typical FOSs are: Forehand topspin behind the table, forehand flip over the table, backhand topspin behind the table and backhand flip over the table. As topspin is always connected to the behind the table position and flip to the over the table position, we will drop the explicit mentioning of the relative position to the table at time of ball contact in the next sections when talking about topspin and flip. Most used for FOS overall was FH topspin (37.4\%), followed by $\mathrm{BH}$ topspin $(29.3 \%$ ) and the $\mathrm{BH}$ flip (22.3\%). FH flip was used least often (10.9\%).

A different frequency order for men and women was obtained when analysed separately. FH topspin is still the most used FOS for both men (35.9\%) and women (39.1\%). But different to the overall order, the second most popular shot for men is the BH flip (27.2\%) and not the BH topspin (23.2\%). For women, BH topspin $(36.1 \%)$ is on second place, followed by $\mathrm{BH}$ flip (17.0\%).

The chi-square test proves a significant relation between sex and the selection of the lateralitytechnique-position combination for the FOS $\left(X^{2}\right.$ $(3, \mathrm{~N}=6654)=264.31, \mathrm{p}<.001)$.

Table 1.

Data sample: match and rally distribution according to match category and sex.

\begin{tabular}{clcccccccc}
\hline & \multicolumn{8}{c}{ Match Category } \\
\cline { 3 - 9 } & & \multicolumn{2}{c}{ Top50 vs Top50 } & \multicolumn{2}{c}{ Top50 vs Over50 } & Over50 vs Over50 & \multicolumn{2}{c}{ Total } \\
\cline { 3 - 9 } & Matches & Rallies & Matches & Rallies & Matches & Rallies & Matches & Rallies \\
\hline Sex & 15 & 1120 & 15 & 1081 & 15 & 1359 & 45 & 3560 \\
& Female & 15 & 1143 & 15 & 1294 & 15 & 1452 & 45 & 3889 \\
& Male & 30 & 2263 & 30 & 2375 & 30 & 2811 & 90 & 7449 \\
\hline
\end{tabular}

Table 2.

Descriptive statistics of FOS laterality-technique-position combination in the two-factor factorial model after excluding marginal shot types.

\begin{tabular}{|c|c|c|c|c|c|c|c|c|c|c|}
\hline \multirow[t]{4}{*}{ Sex } & \multirow[t]{4}{*}{ Match Category } & \multicolumn{4}{|c|}{ Forehand } & \multicolumn{4}{|c|}{ Backhand } & \multirow{4}{*}{$\begin{array}{c}\text { Total } \\
\text { Count }\end{array}$} \\
\hline & & \multirow{2}{*}{\multicolumn{2}{|c|}{$\begin{array}{c}\text { Topspin } \\
\text { behind the table }\end{array}$}} & \multicolumn{2}{|c|}{ Flip } & \multicolumn{2}{|c|}{ Topspin } & \multicolumn{2}{|c|}{ Flip } & \\
\hline & & & & over $\mathrm{t}$ & table & behind & table & over th & table & \\
\hline & & Count & $\%$ & Count & $\%$ & Count & $\%$ & Count & $\%$ & \\
\hline \multirow[t]{4}{*}{ Female } & Top50 vs Top50 & 355 & 34.9 & 96 & 9.4 & 386 & 38.0 & 179 & 17.6 & 1016 \\
\hline & Top50 vs Over50 & 361 & 37.1 & 68 & 7.0 & 360 & 37.0 & 184 & 18.9 & 973 \\
\hline & Over50 vs Over50 & 521 & 44.2 & 85 & 7.2 & 396 & 33.6 & 176 & 14.9 & 1178 \\
\hline & Total & 1237 & 39.1 & 249 & 7.9 & 1142 & 36.1 & 539 & 17.0 & 3167 \\
\hline \multirow[t]{4}{*}{ Male } & Top50 vs Top50 & 383 & 36.5 & 159 & 15.2 & 238 & 22.7 & 268 & 25.6 & 1048 \\
\hline & Top50 vs Over50 & 426 & 36.6 & 165 & 14.2 & 230 & 19.8 & 343 & 29.5 & 1164 \\
\hline & Over50 vs Over50 & 442 & 34.7 & 154 & 12.1 & 342 & 26.8 & 337 & 26.4 & 1275 \\
\hline & Total & 1251 & 35.9 & 478 & 13.7 & 810 & 23.2 & 948 & 27.2 & 3487 \\
\hline \multirow[t]{4}{*}{ Total } & Top50 vs Top50 & 738 & 35.8 & 255 & 12.4 & 624 & 30.2 & 447 & 21.7 & 2064 \\
\hline & Top50 vs Over50 & 787 & 36.8 & 233 & 10.9 & 590 & 27.6 & 527 & 24.7 & 2137 \\
\hline & Over50 vs Over50 & 963 & 39.3 & 239 & 9.7 & 738 & 30.1 & 513 & 20.9 & 2453 \\
\hline & Total & 2488 & 37.4 & 727 & 10.9 & 1952 & 29.3 & 1487 & 22.3 & 6654 \\
\hline
\end{tabular}


Looking at the match categories within each sex, the chi-square tests show a significant relation between the match category and the FOS lateralitytechnique-position combination for women ( $x^{2}$ $(6, N=3167)=26.74, p<.001)$ as well as for men $\left(X_{2}\right.$ $(6, N=3487)=21.86, p=.001)$. Regarding frequencies, it has to be mentioned that for women in the Top 50 vs. Top50 category $\mathrm{BH}$ topspin is most used as FOS, for Top50 vs. Over 50 category $\mathrm{FH}$ topspin and $\mathrm{BH}$ topspin are more or less equal whilst in the Over50 vs. Over50 category the $\mathrm{FH}$ topspin is clearly the most used FOS. For men, in the Over50 vs. Over 50 category the $\mathrm{BH}$ topspin is the second most used FOS, whilst for the other categories the BH Flip is on second position (in all men categories $\mathrm{FH}$ topspin is the most used FOS).

Regarding the position relative to the table, men intend to open the rally more likely over the table than women ( $40.9 \%$ for men vs. $24.9 \%$ for women). The chi-square tests confirmed a significant relation between sex and the position to the table at the point of contact $\left(X^{2}(1, N=6654)=191.68, p<.001\right.$ ). A significant relation is also shown between the match categories and the position relative to the table at the point of contact within each sex (women: $X^{2} \quad(2, N=3167)=7.82, p=.020$, men: $X^{2}$ $(2, N=3487)=6.65, p=.036)$. For the women's categories a trend towards more over the table FOS was recognizable for the categories with more Top50 players (Over50 vs. Over50: $22.2 \%$, Top50 vs. Over50: 25.9\%, Top50 vs. Top50: 27.1\%). Within the men's categories the Top50 vs. Over50 (43.6\%) had the highest percentage of over the table FOS (Over50 vs. Over50: 38.5\%, Top50 vs. Top50: 40.7\%).

After describing what was used as a FOS and in which position to the table it was used, the next important point is to get information when in the rally the FOS was used by the players.

A Mann-Whitney-U-Test was calculated to determine if there were differences in the FOS shot number between women and men. The test proved a statistically significant difference in the shot number between women and men $(U=5307059.00, Z=-2.965$ $, p=.003, r=-.036)$ even though the effect size is very small (Cohen, 1992). The means $\left(\operatorname{mean}_{\text {women }}=\right.$ 2.74, mean $_{\text {men }}=2.83$ ) and grouped medians (grouped median $_{\text {women }}=2.64$, grouped median $\left._{\text {men }}=2.69\right)$ show only a very small difference, too.

Figure 2 shows the distribution of the FOS shot number in a rally separated by gender. In both genders the majority of FOSs were performed with the second, third or fourth shot in a rally (women \#FOS $^{<=4}=96.9 \%$, men $_{\# F O S}<=4=94.9 \%$ ).

A Kruskal-Wallis-Tests indicated first that there is a significant difference in the FOS shot number between the different match categories for women $(H(2)=6.729, p=.035)$, but the post-hoc tests couldn't show any significances. For men no significant difference was found $(H(2)=.404, p=.817)$. This shows that the situations and the moments within a rally when FOS were performed are statistically very similar throughout all male or female match categories respectively, regardless of the FOS player's and the opponent's ranking. Thus, no different tendencies of an earlier or later attacking was found.

By analysing the FOS shot number, we got also the information whether the server (odd shot numbers) or the receiver (even shot numbers) performed the FOS. Figure 3 and Figure 4 show the distribution of the FOS technique for both groups separated for women and men respectively.

In both genders the distribution of the FOSs technique is different whether the server or the receiver performs the FOS. If the server is performing the $\mathrm{FOS}$, topspin $(\mathrm{FH}+\mathrm{BH})$ has a much higher percentage than if the receiver is performing the FOS (women: $91.2 \%$ topspin (FOS by server) vs. $63.7 \%$ topspin (FOS by receiver); men: $74.5 \%$ topspin (FOS by server) vs. 49.1\% (FOS by receiver)). In men's matches, in case the receiving player is performing the FOS, flip technique (50.9\%) is even more often used than the topspin technique. In particular the different use of the $\mathrm{BH}$ flip needs to be mentioned. The chi-square tests proved the significant relation between the FOS technique and FOS by serving/ receiving player for men $\left(X^{2}(3, N=3487)=401.21, p<.001\right)$, women $\left(X^{2}(3, N=3167)=322.67, p<.001\right)$ and overall $\left(X^{2}\right.$ $(3, N=6654)=678.93, p<.001)$. Thus, it can be said that the FOS behaviour of the receiving player is different from the one of the serving player.

After analysing the distribution and incidences of the different FOS variables, we were interested in the respective rally winning probabilities (wp) for the FOS player. Table 3 shows the incidences of won rallies and winning probabilities for the FOS player in our two-factor factorial model.

For our typical FOSs, the winning probabilities showed clear tendencies. The winning probability for the FOS player was always over $50 \%$ when using topspin (FH or $\mathrm{BH}$ ) as FOS with a minimum of $50.4 \%$ in the women's Top50 vs. Top50 category for the $\mathrm{FH}$ topspin and $50.4 \%$ in the men's Top50 vs. Top50 category for the $\mathrm{BH}$ topspin. Using flip as FOS was a disadvantage for the FOS player in two of three categories for men as well as women while executing the flip with $\mathrm{FH}$, and in all categories except the men's Top50 vs. Over 50 category with $\mathrm{BH}$. Table 3 shows that the FH flip is only a "weapon" between two Over50 players as they might not be able to handle the opponents' flip - in contrast to the Top50 players. As the total incidences of the $\mathrm{FH}$ flip technique are the lowest among all used techniques, the winning probabilities might also be influenced by the skill of a certain player and probably have greater fluctuation than the winning probabilities of other techniques with greater incidences. 
Table 3.

Incidences of won rallies and winning probabilities (in \%) for the FOS player for the "typical FOSs" in the two-factor factorial model.

\begin{tabular}{|c|c|c|c|c|c|c|c|c|c|c|c|}
\hline \multirow[t]{4}{*}{ Sex } & \multirow[t]{4}{*}{ Match Category } & \multicolumn{4}{|c|}{ Forehand } & \multicolumn{4}{|c|}{ Backhand } & \multicolumn{2}{|c|}{ Total } \\
\hline & & \multicolumn{2}{|c|}{ Topspin } & \multicolumn{2}{|c|}{ Flip } & \multicolumn{2}{|c|}{ Topspin } & \multicolumn{2}{|c|}{ Flip } & \multirow[b]{3}{*}{ Count } & \multirow[b]{3}{*}{$\%$} \\
\hline & & \multicolumn{2}{|c|}{ behind the table } & \multicolumn{2}{|c|}{ over the table } & \multicolumn{2}{|c|}{ behind the table } & \multicolumn{2}{|c|}{ over the table } & & \\
\hline & & Count & $\%$ & Count & $\%$ & Count & $\%$ & Count & $\%$ & & \\
\hline \multirow[t]{4}{*}{ Female } & Top50 vs Top50 & 179 & 50.4 & 45 & 46.9 & 200 & 51.8 & 89 & 49.7 & 513 & 50.5 \\
\hline & Top50 vs Over50 & 211 & 58.4 & 24 & 35.3 & 190 & 52.8 & 77 & 41.8 & 502 & 51.6 \\
\hline & Over50 vs Over50 & 294 & 56.4 & 43 & 50.6 & 201 & 50.8 & 77 & 43.8 & 615 & 52.2 \\
\hline & Total & 684 & 55.3 & 112 & 45.0 & 591 & 51.8 & 243 & 45.1 & 1630 & 51.5 \\
\hline \multirow[t]{3}{*}{ Male } & Top50 vs Top50 & 226 & 59.0 & 55 & 34.6 & 120 & 50.4 & 122 & 45.5 & 523 & 49.9 \\
\hline & Top50 vs Over50 & 232 & 54.5 & 74 & 44.8 & 130 & 56.5 & 175 & 51.0 & 611 & 52.5 \\
\hline & Over50 vs Over50 & 259 & 58.6 & 79 & 51.3 & 193 & 56.4 & 164 & 48.7 & 695 & 54.5 \\
\hline
\end{tabular}

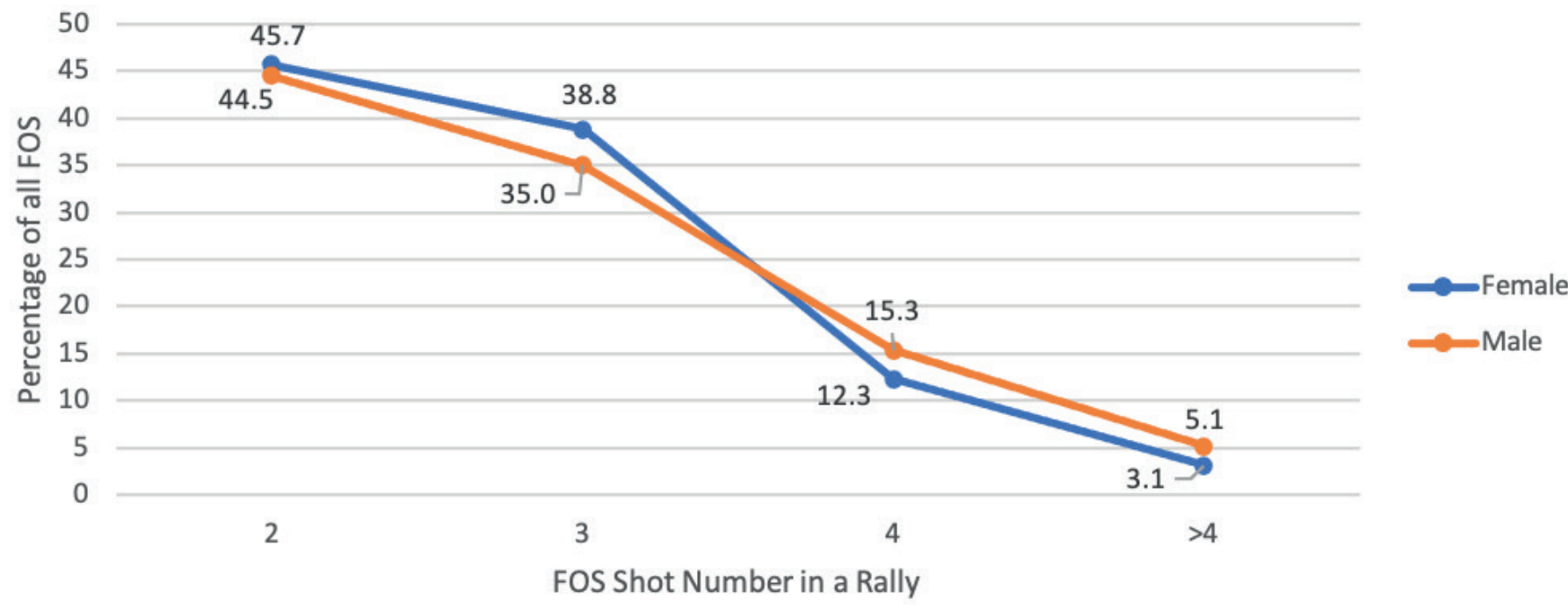

Figure 2. Distribution of the FOS Shot Number in a Rally.

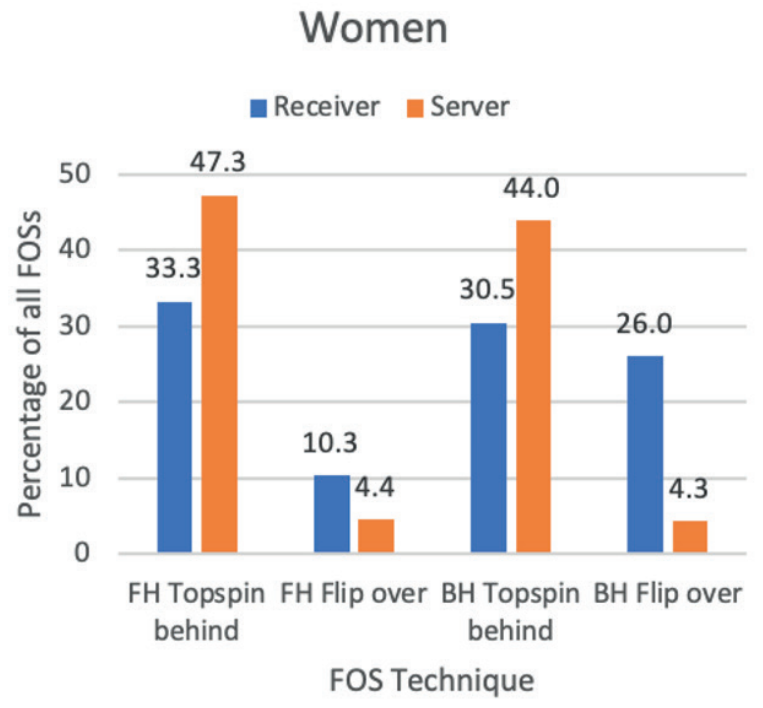

Figure 3. Distribution of FOSs separated by server/receiver for women.

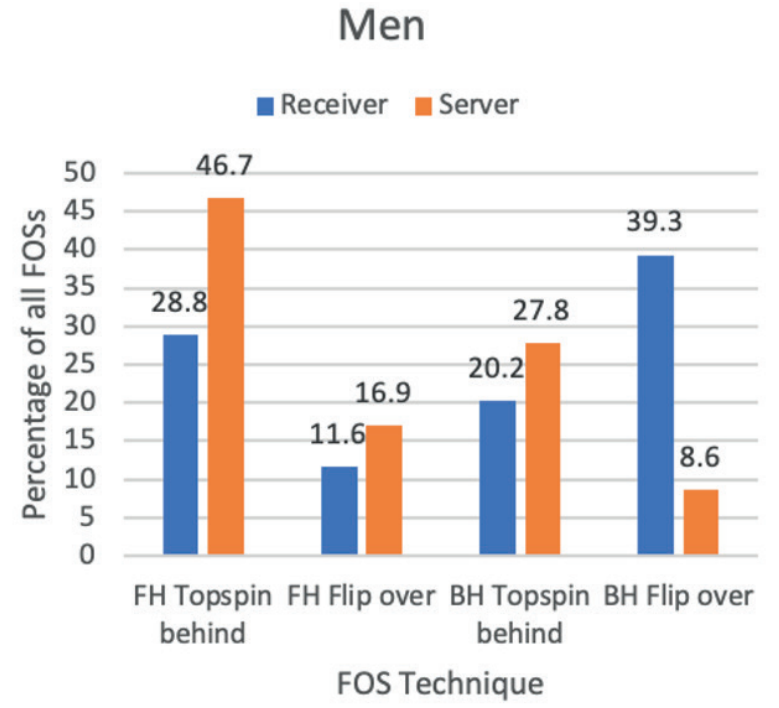

Figure 4. Distribution of FOSs separated by server/receiver for men. 


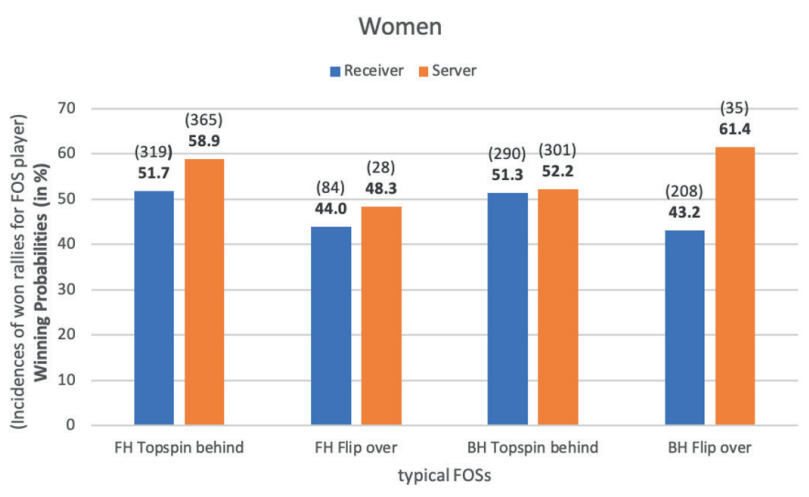

Figure 5. Incidences of won rallies and winning probabilities for the FOS player using the typical FOSs separated by serving or receiving player is performing the FOS for women's matches.

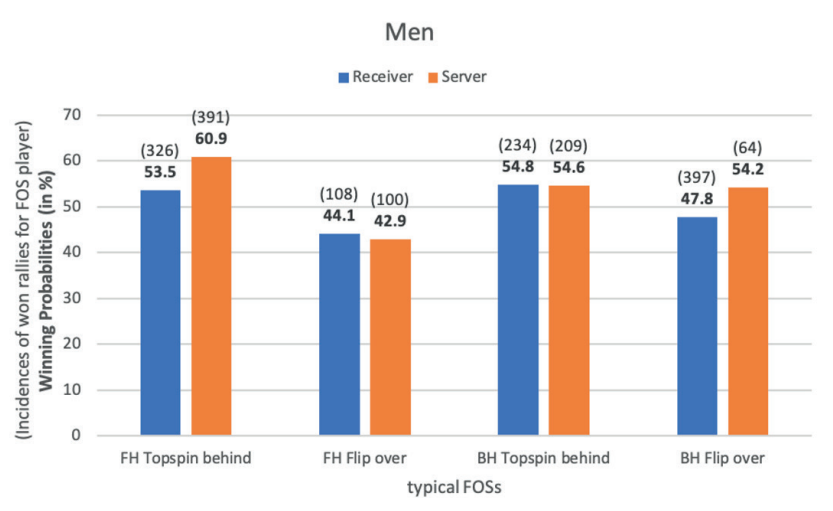

Figure 6. Incidences of won rallies and winning probabilities for the FOS player using the typical FOSs separated by serving or receiving player is performing the FOS for men's matches.

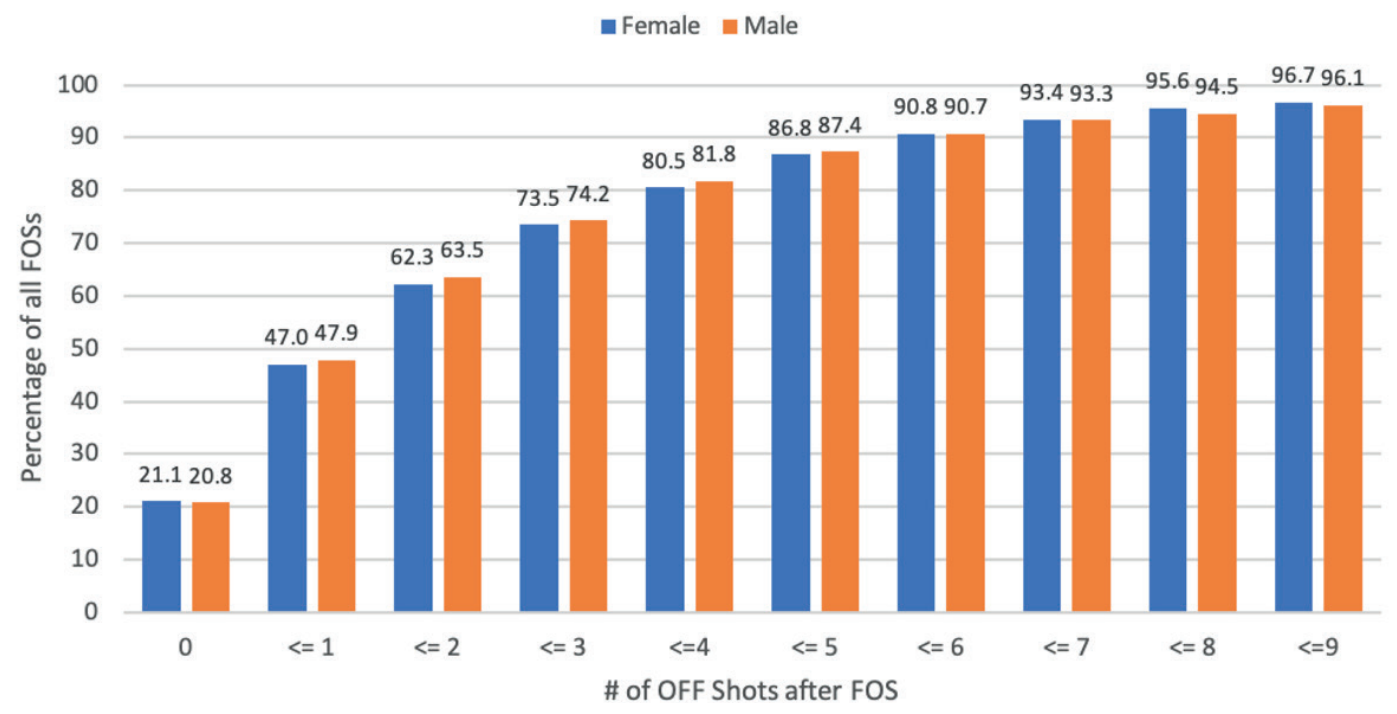

Figure 7. Distribution of the number of offensive shots after the FOS.

Figure 5 (for women) and Figure 6 (for men) show the incidences of won rallies and winning probabilities for the FOS player using the typical FOSs separated by serving or receiving player is performing the FOS.

Topspin with $\mathrm{FH}$ or $\mathrm{BH}$ as FOS was always an advantage for the server as well as for the receiver. In contrast, using the flip was always a disadvantage except when the server could perform a BH flip as FOS (wp BH flip mserver $=54.2 \%$, wp BH flip wserver $=61.4 \%$ ).

The biggest difference in FOS winning probabilities between the serving and receiving player were found for the $\mathrm{FH}$ topspin (wp FH topspin mReceiver $=53.5 \%$ vs. $w p F H$ topspin mserver $=60.9 \%$, wp $\mathrm{FH}$ topspin wReceiver $=51.7 \%$ vs. wp $\mathrm{FH}$ topspin ${ }_{\text {wserver }}$ $=58.9 \%$ ) and the BH flip (wp BH flip meeceiver $=47.8 \%$ vs. $\mathrm{wp} \mathrm{BH}$ flip mserver $=54.2 \%$, wp BH flip wReceiver $=43.2 \%$ vs. wp BH flip wserver $=61.4 \%$ ) in both genders with the higher winning probabilities for the serving player.

The FOS is arguably a crucial moment in a rally, but the advantage/disadvantage by performing the FOS might be neutralized in longer rallies. Figure 7 shows the distribution of the number of offensive shots after the FOS in a rally.
Over $60 \%$ of all rallies (women: $62.3 \%$; men: $63.5 \%$ ) are finished with a maximum of two offensive shots after the FOS, and almost three quarters are finished with three or less shots after the FOS (women: $73.5 \%$; men: $74.2 \%)$.

To get a better indication of the direct impact of the FOS, the sample was additionally split into two subsamples. The upper limit for the direct impact of the FOS was derived by an inspiration of the first phase of the Three-Phase-Model approach (Wu \& $\mathrm{Li}$, 1992) with the FOS as starting point. As direct impact we defined rallies which finished latest with the follow up attack after the FOS of the FOS player (including a possible mistake of the opponent with the following shot) which leads to the first, the "direct impact of FOS" subsample. The second ("no direct impact of FOS") subsample includes all remaining rallies with more offensive shots after the FOS. Figure 8 and Figure 9 show the incidences of rallies with a direct impact or no direct impact of the respective FOSs. The figures show the same trend, regardless of the used FOS. More rallies are finished with a direct impact of the respective FOS. 
When talking about the intention the FOS player should have, Figure 10 and Figure 11 are clearly showing that it was more successful for the FOS player if a rally was finished with a direct impact of the FOS. Only the rallies in women's matches with FH flip as FOS showed a higher winning probability for the FOS player in the longer rallies compared to the "direct impact of FOS" rallies (wp FH flip wDirectlmpact $=44.1 \%$ vs. wp FH flip wNoDirectlmpact $=47.6 \%$ ).

\section{Discussion}

This study aimed to give a better understanding of the FOS in a rally in top level table tennis. For that purpose, FOS shot number, FOS laterality, FOS technique and FOS position towards the table at the point of contact have been analysed while taking

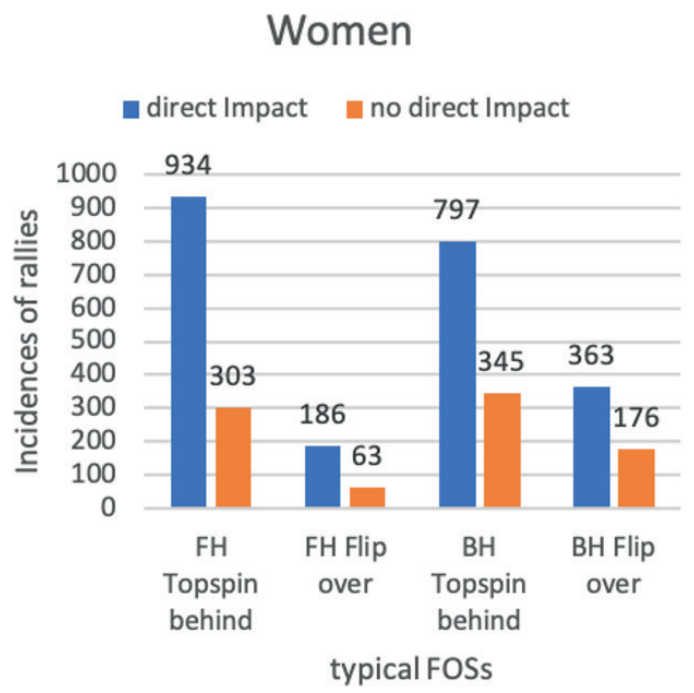

Figure 8. Incidences of typical FOSs separated by impact of FOS for women. into account the influence of sex and the respective player/match category. Additionally, the winning probabilities for the FOS player have been analysed. The inter-observer reliability tests for all variables showed excellent inter-rater agreements. For the identification of the FOS and the laterality of the FOS an almost perfect agreement was expected as the differences between defensive and offensive shots and FH/BH can be identified easily. Although assigning the levels of the other observed variables for the FOS (technique and position) is not trivial, still a high agreement between experienced coaches was expected as only very few situations, e.g. short BH topspin movements vs. BH flip after a half-long ball, might result in different value assignments. But in most cases, the techniques and the position relative to the table is clearly recognizable by (experienced) observers.

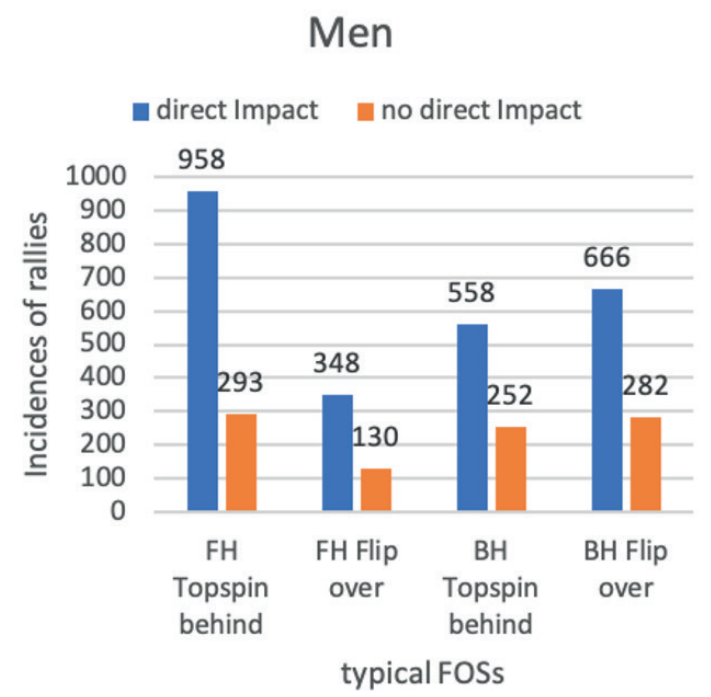

Figure 9. Incidences of typical FOSs separated by impact of FOS for men.

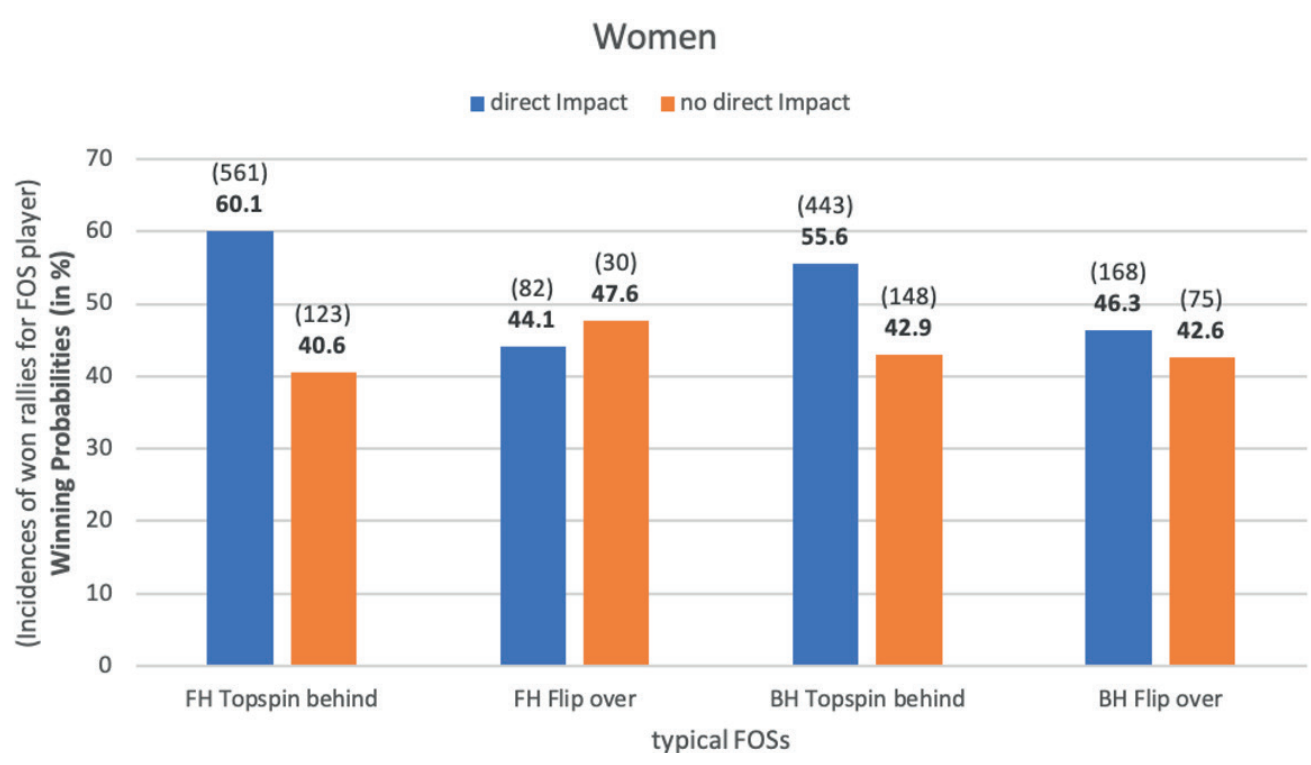

Figure 10. Incidences of won rallies and winning probabilities for the FOS player using the typical FOSs separated by direct or no direct impact of the FOS for women's matches. 


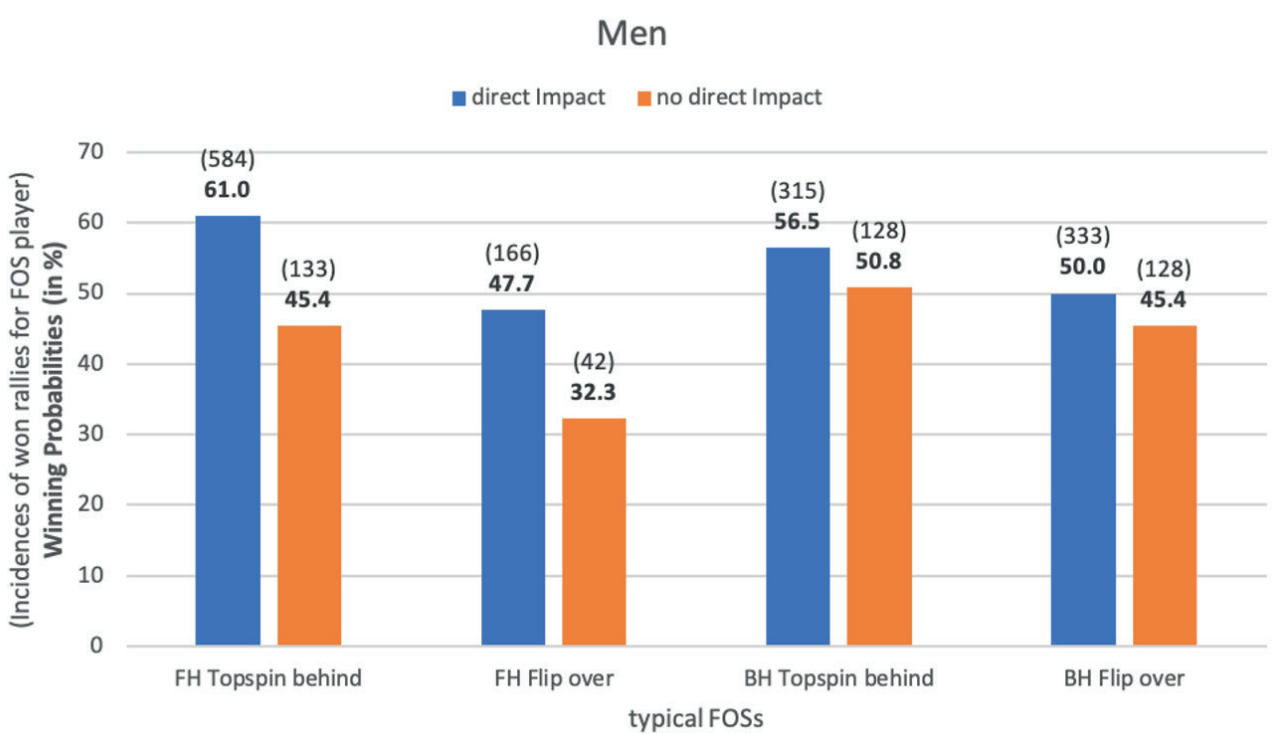

Figure 11. Incidences of won rallies and winning probabilities for the FOS player using the typical FOSs separated by direct or no direct impact of the FOS for men's matches.

The results of this study revealed that there are four typical FOSs covering $98 \%$ of all FOSs. The women's more frequent use of backhand as FOS might be caused by the fast and close to the table (and because of that often more backhand oriented) playing style of women compared to men.

The FH topspin is the most used FOS in all men's match categories and in two of three women's categories. Only in the women's Top50 vs. Top50 category the $\mathrm{BH}$ topspin is more often used than the FH topspin. Overall, female players prefer the topspin technique (FH and $\mathrm{BH}$ topspin clearly on first and second place covering together more than $73 \%$ of FOSs in all categories) over the flip technique. The flip technique is more often used in men's compared to women's matches leading to a significantly different distribution of the techniques between men and women. Especially the more frequent use of the backhand flip in the men's matches (which is tantamount to more over the table FOS) is probably explained by a different serve and receive game in the different genders. Men are more likely serving short - which is confirmed by the study of Djokic et al. (2020) - to avoid a direct full swing topspin of the opponent whilst - following Zhang and Zhou (2017)women don't seem to be afraid to serve or push with the receive more frequently half long or long because the opponents opening topspin can be controlled or even countered. This might be based on differences in physical capabilities between men and women which lead to a possibly stronger FOS topspin in the men's game which opponents are afraid of.

A similar average FOS number for men and women (grouped median $_{\text {women }}=2.64$, grouped median $_{\text {men }}$ $=2.69$ ) with the different FOS behaviour explained before supports the explanation that the FOS might be highly affected or rather controlled by the serve as not too much short-short game is going on for both genders. To prove this hypothesis, additional research on the shot(s) prior to the FOS needs to be done.

The different FOS behaviour between server and receiver might also be explained by the receiving game of the majority of players, especially for men. The popularity of the $\mathrm{BH}$ banana flip directly as receive might be one reason. The difficulty to receive really short a second one. It seems that if the receiver doesn't attack directly, but plays a defensive push, the server can (or needs to) attack that push very often with a topspin. This can happen due to a failed short push which went half long or long but also due to the conscious decision to receive with an aggressive long push. The second option seems to get more and more popular. With a controlled long push, the receiver provokes a topspin with more spin from behind the table, which might also be easier to control. The attempt to push short involves always the risk of a qualitatively bad or too high short ball which can be easier killed than a long push loaded with backspin.

Regarding the winning probabilities, explanations for certain results are very hard to give as so many factors come into play. Noticeable is that the winning probabilities for topspin techniques as FOS were always $>50 \%$ and always bigger than the winning probabilities for the flips.

Using the flip as FOS was a disadvantage for the FOS player in nine out of twelve cases (Table 3). Only the FH flip in the women's and men's Over50 vs Over50, as well as the BH flip in the men's Top50 vs Over50 category showed winning probabilities $>50 \%$. There may be diverse reasons why the flip as FOS is not an advantage. For example, in the male Top50 vs Top50 category (wpFH flip $=34.6 \%$ ), the non-FOS player 
might be able to counter the flip with high quality. The flip technique itself might be the problem in that category as it is simply not strong enough in terms of spin (opponent can control the top-/sidespin) and speed (opponent is fast enough and has very good anticipation) which might be different in categories with lower ranked players involved.

Although it can be stated that topspin is the more successful FOS than flip, exclusively using topspin as FOS is not an option. Using flip (especially as a receiver) might be highly influenced by the opponent's serve which might give no possibility to topspin and at the same time makes it very difficult to play a good short ball. Thus, players are almost forced to play a flip in those situations.

Following the analysis of the post FOS shot number and the respective winning probabilities, the intention for the FOS should be to finish the rally with his/her follow up shot. Especially when using topspin as FOS, the winning probabilities for the FOS player were way higher in the rallies finished quickly after the FOS compared to the longer rallies. This means, when opening the rally with a topspin, the FOS player should try to kill the ball with his/her next shot at the latest. Otherwise, the advantage will decrease noticeably (decrease for women matches at 19.5\% (FH topspin) and $12.7 \%$ (BH topspin), decrease for men's matches at $15.6 \%$ (FH topspin) and 5.7\% (BH topspin)). For the flip as FOS, no clear trend could be found (big decrease for FH flip in men's matches, but even an increase for FH flip in women's matches; decreases for $\mathrm{BH}$ flip $<5 \%$ for both genders). Thus, no general advice for the tactical behaviour after the flip as FOS can be given based on the study's results as it seems to be a more individual consideration whether and when the FOS player is successful with the flip as FOS.

Following the findings of our study, some practical suggestions for players and coaches can be given: As flip was a disadvantage and topspin was an advantage overall, the importance of a good quality short push instead of opening the rally with a flip should be taken into account in the daily work. Closing the rally with the follow up attack after a player could take the initiative with a topspin is a second important finding for players and coaches not only for competitions, but also in the design of competition-like exercises in training.

Some limitations of the study need to be acknowledged. First, the FOS behaviour and the FOS winning probabilities are highly influenced by the placement and quality of the shot prior to the FOS which is not analysed in this study. This information could help to identify e.g. after which placement certain FOSs are (successfully) performed. Second, the world rankings of the players - especially in first round matches including wild card players - show larger variance. Thus, although players belong to the same player category, there might be a difference in the level of skills which might have an influence on the FOS behaviour especially on the winning probabilities of certain techniques.

Despite these limitations, the conceptual advantages of the introduced process model for a table tennis rally could be clearly shown. Tactical behaviour for the first offensive shot could be analysed without any dependency on a specific shot number. The results for the FOS number underlined the necessity of such a shot-number-independent approach as the FOS number was spread over a range of shot numbers. Moreover, ambiguities in shotnumber-based approaches - a third shot may be a defensive short push as well as an all-in attacking shot - speak as well in favour of the presented, shot number independent FOS analysis.

\section{Conclusion}

This study provides a first overview of the FOS behaviour in elite table tennis using a new shotnumber-independent approach. The detailed technical/tactical analyses of the FOS behaviour, including the analysis of the winning probabilities gives more precise knowledge about the structure of the sport. The reliable information about different FOS behaviour for men and women or for the serving and receiving player respectively, the differences between match categories and the differences of winning probabilities could lead to practical implications for training and competition and also to adaptions in the tactical education in the development of (young) athletes.

\section{Funding \& Conflict of interests}

No funding was provided for the conductance of this study. The authors declare no conflict of interests.

\section{References}

Clemett, C. (2010). Evolution of the Laws of Table Tennis and the Regulations for International Competitions [PDF file]. Retrieved April 27, 2020, from https:// www.ittf.com/wp-content/uploads/2016/10/ ColinEvolution.pdf

Cohen, J. (1988). Statistical power analysis for the behavioral sciences. L. Erlbaum Associates.

Cohen, J. (1992, Jul). A power primer. Psychol Bull, 112(1), 155-159. https: / /doi.org/10.1037/ /0033-2909.112.1.155

Djokic, Z., Malagoli Lanzoni, I., Katsikadelis, M., \& Straub, G. (2020, 06/30). Serve analyses of elite European table tennis matches. International Journal of Racket Sports Science, 2(1). https:// journal.racketsportscience.org/index.php/ijrss/ article/view/22 
Djokic, Z., Munivrana, G., \& Levajac, D. (2017). Match analyses of final game of Men's Team European Championships 2014 - Portugal vs. Germany. In M. Kondric, X. Zhang, \& D. Xiao (Eds.), Science and Racket Sports V (pp. 113-121). Soochow University Press.

Fritz, C. O., Morris, P. E., \& Richler, J. J. (2012, Feb). Effect size estimates: current use, calculations, and interpretation. J Exp Psychol Gen, 141(1), 2-18. https://doi.org/10.1037/a0024338

Fuchs, M., Liu, R., Malagoli Lanzoni, I., Munivrana, G., Straub, G., Tamaki, S., Yoshida, K., Zhang, H., \& Lames, M. (2018, Dec). Table tennis match analysis: a review. Journal of Sports Sciences, 36(23), 26532662. https://doi.org/10.1080/02640414.2018.14500 73

International Olympic Committee. (2016). Conditions of Participation - National Olympic Committee Rio 2016. Retrieved 14th January from http://naaz. co.zw/Portals/0/PropertyAgent/458/Files/164/ Rio\%202016_Conditions\%20of\%20Participation_ National\%200lympic\%20Committee.pdf

International Table Tennis Federation. (2021). 2021 ITTF Table Tennis World Ranking. Retrieved 14th January from https://www.ittf.com/2021-ittf-table-tennisworld-ranking/

Koo, T. K., \& Li, M. Y. (2016, Jun). A Guideline of Selecting and Reporting Intraclass Correlation Coefficients for Reliability Research. J Chiropr Med, 15(2), 155163. https://doi.org/10.1016/j.jcm.2016.02.012

Küneth, T. (2020). The transition from celluloid to plastic balls. International Table Tennis Federation. Retrieved April 27 from https://www. ittf.com/2020/01/28/transition-celluloid-plasticballs/

Lames, M., Fuchs, M., \& Wenninger, S. (2018). Wettkampfdiagnostik im Tischtennis. In BISpJahrbuch : Forschungsförderung 2016/17 (pp. 8591). https://www.bisp.de/SharedDocs/Downloads/ Publikationen / Jahrbuch / Jahrbuch_2016_2017_ Gesamt.pdf?__blob=publicationFile\&v=2, http:// my.page2flip.de/2895682/14185088/14185117/ html5.html\#/86
Malagoli Lanzoni, I., Di Michele, R., \& Merni, F. (2014). A notational analysis of shot characteristics in toplevel table tennis players. European Journal of Sport Science, 14(4), 309-317. http://search.ebscohost. com/login.aspx?direct=true \& db=sph\&AN=9586170 $6 \&$ site $=$ ehost-live

Newell, K. M. (1986). Constraints on the Development of Coordination. In N. G. Wade \& H. T. Whiting (Eds.), Motor Development in Children: Aspects of Coordination and Control (pp. 341-360). Matinus Nijhoff, Dordrecht.

Tamaki, S., Yoshida, K., \& Yamada, K. (2017, Jan 01). A Shot Number Based Approach to Performance Analysis in Table Tennis. J Hum Kinet, 55, 7-18. https://doi.org/10.1515/hukin-2017-0002

Townsend, W.(2017). Tuttifruttitabletennisterminology. International Table Tennis Federation. Retrieved April 27 from https://www.ittf.com/2017/09/10/ tutti-frutti-table-tennis-terminology/

Wu, H., \& Li, Z. (1992). Research on technical diagnosis method for table tennis players. International Journal of Table Tennis Sciences, 1, 99-103.

Zhang, H., Liu, W., Hu, J. J., \& Liu, R. (2013). Evaluation of elite table tennis players' technique effectiveness. Journal of Sports Sciences, 31(14), 1526-1534. http:/ / search.ebscohost.com/login.aspx?direct=true \&db= sph \&AN=90464904\& site=ehost - live

Zhang, H., \& Zhou, Z. (2017, 2017/11/02). An analytical model of the two basic situation strategies in table tennis. International Journal of Performance Analysis in Sport, 17(6), 970-985. https:/ /doi.org/10. $1080 / 24748668.2017 .1415071$ 